\title{
An EDC Information EDC Visualization System in the Clinical Trials
}

\author{
Yujia Kong, Fuyan Shi \\ Kong Yujia, An Hongqing, Bian Weiwei and Shi Fuyan* \\ School of public health, Weifang Medical College, Shandong, China \\ Corresponding author: Shi Fuyan
}

Keywords: EDC; Clinical trials; information

\begin{abstract}
It is essential to popularize the application of EDC in clinical trials. To provide reference for the standard management of clinical trials and to update pharmaceutical R\&D industry on electronic data capture( EDC) in drug clinical trials. The advantages and development trends were summarized. The EDC design functions of electronic case report form should be strengthened in China and the connection between EDC and ADR may be the core of future studies.
\end{abstract}

\section{Introduction}

Randomization design phase of clinical trial research clinical investigators must face a problem. Randomized grouping method studies have shown that the traditional randomization methods are increasingly unable to meet demand.Dynamic random method and central randomization system frequently mentioned is considered to be an important means to control the prognostic factors.Dynamic randomized algorithms, minimization and dynamic balanced randomization method different emphases in the control of the prognostic factors. The balance under Minimization in dealing with the same importance of various prognostic factors is better.The balance of dynamic balanced randomization method in dealing with the hierarchy of importance of various prognostic factors is better.How effective combination of the two full play to their superiority, to obtain the optimized random effect, it is worth the statistics further explore. The aim of the clinical trial data management, data from subjects, complete and accurate record.Record of all operations involved in each step of the data management. Which is conducive to supervise and inspect the quality of data and test the implementation process.

The role of statistics in clinical trials has been recognized by the majority of clinicians .Whether the study design, data collection, or statistical analysis, statistically has infiltrated all aspects of clinical trials. Standardization of clinical trials of the building is through the implementation of specific standardized measures in various stages of clinical trials, thus ensuring the authenticity and reliability of the results of clinical trials. Domestic and foreign government departments have recognized the importance of standardized, have introduced a number of regulations, the principle requirements of the operation of the clinical trials. ndustry Association also announced a series of clinical trials, standardized instructions, the industry standard for the implementation of specific clinical trials.Domestic and international use of Electronic Data Capture (EDC) has developed a lot of electronic data management system.How to scientifically manage the clinical data acquisition, entry, verification, lock, export, and work a good attempt.

\section{Model construction and system implementation}

\section{Data analysis}

The first task of constructing a EDC Visualization platform is to understand the feature of the data. The ADR data were obtained from two sources: the drug-ADR combinations included in drug instructions and those detected by ADR monitoring agencies. Some drug-ADR combinations were common between the two groups (Figure 1). 


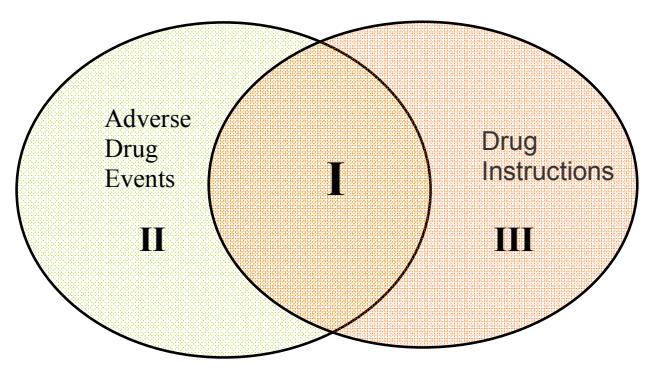

Figure1. Three types of Drug-ADR combinations

Four tables were needed for this study. The ADEs table, a total of 4,404 Drug-ADR combinations including 656 common drugs, was provided by China Jiangsu Province ADR Monitoring Center. The data fields included drug code, ADRs code, and event frequency. 7,150 Drug-ADR combinations were retrieved from the Internet for the establishment of drug instructions table (The data fields include drug code and ADRs code). Due to the differences of data acquisition, the record numbers of the above two tables were not consistent. The third table was the drug basic information table (The data fields include drug code, name, and type). The forth table was ADRs coding table(The data fields include ADRs code, name, and affected organs), mainly from the World Health Organization Adverse Reaction Terms(WHO-ART).

\section{EDC Visualization legends design}

Three different legends were designed to distinguish the three types of cases in Table 1.

Table 1. Instructions of three legends

\begin{tabular}{ll} 
Type & \multicolumn{1}{c}{ Note } \\
\hline CASE I & $\begin{array}{l}\text { Solid circle, indicating that the ADR of } \\
\text { one specific drug was stated in drug } \\
\text { instructions and also actually detected. } \\
\text { Solid rectangle, indicating that the ADR } \\
\text { of one specific drug was actually } \\
\text { detected but not stated in drug } \\
\text { instructions. }\end{array}$ \\
CASE II & $\begin{array}{l}\text { Hollow dashed circle, indicating that the } \\
\text { ADR of one specific drug was stated in } \\
\text { drug instructions but not actually } \\
\text { detected. }\end{array}$
\end{tabular}

In table 1, as the case I and case III both denote all ADRs stated in drug instruction of a drug, they share the same shape, circle. The case II uses another shape, rectangle, to denote those ADRs not stated in drug instruction. Due to the frequency of the case I and case II is more than zero, solid shape is used. Contrarily, hollow dashed circle is choosing for the shape of the case III since its frequency of equals zero.

\section{EDC Visualization model design}

As one drug has many ADRs, it is one-to-many relationship between them. The Ferris wheel style is just fit to this feature. The overall structure is circular, with the drug as the center connected by the legends of adverse reactions with equal radii. The EDC Visualization model is shown in Figure 2. 


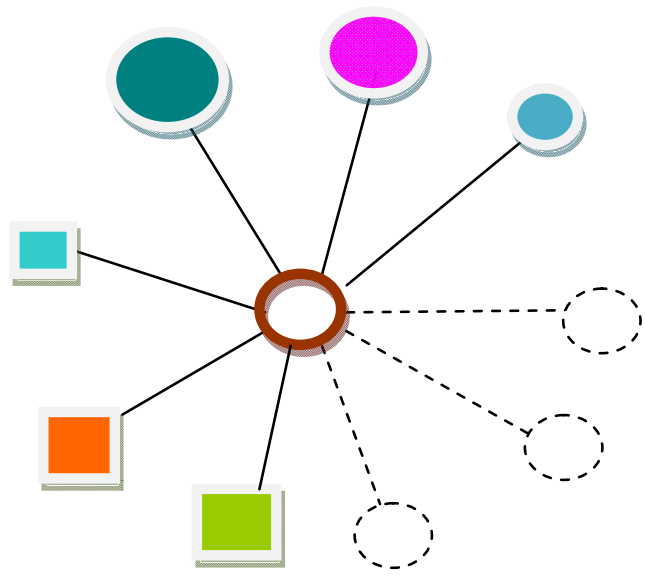

Figure 2. Ferris wheel model

The model met the following requirements:

(1) Different legends to distinguish the three different cases (See table 1).

(2) Graphics with different size to distinguish the frequencies of ADRs. The radii and frequencies were positively correlated, and the legends were arranged clockwise with descending size under the same case.

(3) Different colors to distinguish different types of ADRs. The legend and its name label shared the same color.

(4) Dotted or solid lines to distinguish whether drug-adverse reaction combinations were actually detected.

(5) Certain mathematical model for establishing positional relationships among all legends to ensure overall symmetry and aesthetics (See Figure 3 ).

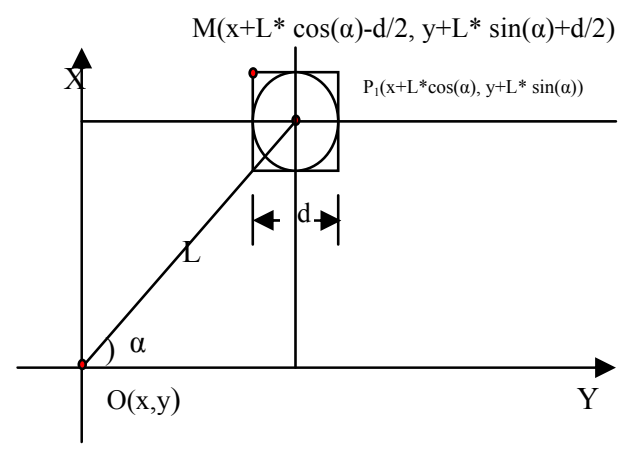

Figure 3: Mathematical model for positional relationships of legends The mathematical model design is as follows:

(1) Construct a coordinates system. Point $O$ denotes as a drug instance and locates in the center of java draw panel. $(x, y)$ is the coordinate value of $O$ and it is a fixed value in this system.

(2) Point $P_{l}$ represents the first ADR of the drug and its coordinate value is $(x+L * \cos (\alpha), y+L * \sin (\alpha))$. $L$ is a fixed value and represents the length of $O P_{1} . \alpha$ is the angle between horizontal ordinate $O Y$ and $O P_{1}$ and it can be calculated from 360/n ( $n$ is the total amount of ADRs of the drug). So the coordinate value of the $i$ th Point $P_{i}$ can be got by $(x+L * \cos (i * \alpha), y+L * \sin (i * \alpha)), i=1,2,3,, n$.

(3) In this system, shape (circle or square) with point $P_{i}$ as center is selected to indicate an ADR instance. The diameter of the shape is $d$. The size of $d$ is determined by the frequency of ADRs and it can be calculated by $\pi(d / 2)^{2} / c$, where $c$ is a constant.

(4) In the draw function of java, coordinate $(x, y)$ indicates the location of the upper-left corner of the shape, and all values are measured in pixel units. Therefore, while drawing a shape, $\mathrm{M}_{(x+L * \cos (i * \alpha)-d / 2, y+L * \sin (i * \alpha)+d / 2)}$ is the coordinate value of draw functions. 


\section{Area of statistical information design}

Compared to graph, text can provide more quantitative information description. In EDC platform, we embed a textual area of statistical information about ADRs of three cases. These information included ADR name, frequency, whether stated in drug instruction and PRR value. Frequency was the total amount of a target ADR of this drug, which has been detected by Jiangsu Province ADR Monitoring Center of China during two years.

PRR is a popular statistic method for ADR signal detection based on disproportionality theory . The formula of PPR method is as follows:

$$
\operatorname{PRR}=\frac{\frac{a}{a+b}}{\frac{c}{c+d}}(1)
$$

Where, a, b, c and d can be found in table 2 .

Table 2.Two-by-two contingency table

\begin{tabular}{lcc}
\hline & Candidate ADR & All other ADRs \\
\hline Candidate drug & $\mathrm{a}$ & $\mathrm{b}$ \\
All other drugs & $\mathrm{c}$ & $\mathrm{d}$ \\
\hline
\end{tabular}

PRR value represents the disproportion degree of a candidate Drug-ADR combination. According to the criteria of ADR signal detection, when $a \geq 2$ and $P R R \geq 3$, the corresponding combination will be considered as a suspect sign. Thus, we highlighted such a suspect signal with "*” before the $P R R$ value in this platform.

\section{Conclusion}

In this study, we put forward a prototype system for EDC information visualization. But to be truly applied to the practice, there are many aspects should be improved in the further work.

First of all is the data problem. It only contains two years ADEs data of one province in china and the drug instruction data was collected from the Internet. Therefore, this platform had yet not provided very sufficient and accurate information for users. In next work, we will enhance the data quantity and data quality of this system.

\section{Acknowledgments}

This paper was supported by the Fund (Funding information: K11QC1011)

\section{REFERENCE}

[1] Jon Duke, Anthony Faiola, and Hadi Kharrazi.A novel visualization tool for evaluating medication side-effects in multi-drug regimens. Proceedings of the 13th International Conference on Human-Computer Interaction,Part IV: Interacting in Various Application Domains,(2009)

[2] Damien McAullay, Graham Williams,Jie Chen,A delivery framework for health data mining and analytics.Proceedings of the Twenty-eighth Australasian conference on Computer Science,Newcastle, Australia,Jan. (2005)

[3] .Gert Van Valkenhoef, Tommi Tervonen, Tijs Zwinkels, Bert De Brock, Hans Hillege.ADDIS : A decision support system for evidence-based medicine.Decision Support Systems,2,55(2013)

[4] Edwards IR, Aronson JK. Adverse drug reactions: definitions, diagnosis, and management. Lancet (2000)

[5] Evans S J W, Waller PC, Davis S. Use of proportional reporting ratios (PRRs) for signal generation from spontaneous adverse drug reaction reports. Pharmacoepidemiol Drug Safe(2001) 